El Guiniguada • Revista de investigaciones y experiencias en Ciencias de la Educación

\title{
Algunas RefleXiones EN TORNO AL BLENDED LEARNING
}

\section{REFLECTIONS ON BLENDED LEARNING}

\author{
Ana Llamazares Carballo \\ HU University of Applied Sciences Utrecht
}

Recibido: 28/03/2015/Aceptado: 15/04/2015

\section{RESUMEN}

El presente trabajo aclara el significado de los términos E-learning, blended learning, Flipping the Classroom, BYOUD, TPACK, su relevancia para la educación en el futuro y posibles obstáculos y retos para su implementación.

Palabras clave: Educación, TIC, aprendizaje combinado, clase invertida, Conocimiento Técnico Pedagógico del Contenido.

\section{ABSTRACT}

This article reflects on the significance of terms like E-learning, blended learning, Flipping the Classroom, BYOUD, TPACK and its consequences for teaching in the future. The possible benefits, obstacles and actions to be taken for its success will be described in the following paper.

Keywords: Education, ICT, blended learning, flipped classroom, TPACK. 


\section{INTRODUCIENDO NUEVOS TÉRMINOS}

Los Ministerios de Educación y Economía holandeses, conjuntamente con los Consejos de la Educación Primaria y Secundaria lanzaron el Proyecto Leerling 2020 (El alumno en el 2020). En él se destaca la urgente necesidad de una educación excelente y personalizada debido a la posición preferencial de Holanda en una economía globalizada. El proyecto aspira a fomentar el desarrollo de una educación a medida, un aprendizaje activo centrado en el alumno, con una organización de la educación eficiente (cumplimiento de los objetivos con el mínimo de costes) y transparencia acerca de los resultados obtenidos. El uso de las TIC se considera clave para poder realizar estas aspiraciones y preparar al alumno para la sociedad del siglo XXI. Dejando aparte los aspectos que se centran en la infraestructura educativa, por ejemplo, el uso del hardware adecuado, las conexiones Wifi, el uso de software para el registro de alumnos, etc., y concentrándose exclusivamente en lo que ocurre en el aula a la hora de impartir los contenidos, aparece una terminología que presenta términos como, por ejemplo, E-learning, blended learning, Flipping the Classroom, BYOUD, TPACK. Estos términos requieren una aclaración para que futuros profesores y todos aquellos relacionados con la enseñanza entiendan de dónde vienen y por qué son importantes.

\section{Definiciones}

Indagando sobre el significado de cada uno de los términos, las definiciones encontradas son más o menos amplias, empleadas a veces de manera aleatoria. Varios estudiosos han hecho intentos de aclarar la terminología, imprescindible para poder entenderse bien cuando se alude a ella. Empezando por E-learning, se podría decir que consiste en la educación a través de un entorno digital conectado a una red en línea. En esta modalidad de la educación se utilizan las herramientas tecnológicas y de la información para realizar el aprendizaje a distancia, de manera no presencial, a través de una plataforma virtual, es decir, un espacio virtual cerrado y creado específicamente para dicho aprendizaje.

Las definiciones de Blended Learning, o B-learning, en un principio contrastan con E-learning en el aspecto de la presencialidad. Hablan de la combinación integrada de la educación tradicional y el aprendizaje online, de una mezcla de aprendizaje con y sin apoyo tecnológico, de la combinación de herramientas y medios en un ambiente E-Learning y, por último, la combinación de estrategias didácticas, independientemente del uso de las tecnologías (Oliver y Trigwell, 2005). Las definiciones más recientes restan importancia al empleo de los medios para centrarse en el aprendiente y su percepción del proceso de aprendizaje. Esta interpretación del término 
mezcla el E-learning y otras maneras de enseñanza prestando además atención a la distribución de contenidos, formas de comunicación, estrategias didácticas y entornos de aprendizaje en relación a los procesos de aprendizaje. Esta aclaración refleja la complejidad del diseño de los procesos de aprendizaje: blended learning, no es hacer lo de siempre en otro entorno, el virtual, esta vez. Implica rediseñar tanto la práctica educativa como el entorno de aprendizaje y requiere de ciertas competencias específicas para monitorizar los procesos de aprendizaje (Fransen, 2006). Cómo hacerlo se verá más adelante.

Bergmann y Sams desarrollaron alrededor del 2006 el concepto de lo que luego vino a llamarse la Flipped Classroom ${ }^{1}$, es decir, la clase invertida o la clase al revés. Para solventar el problema de falta de asistencia a clase de alumnos que vivían en zonas remotas, estos docentes de educación secundaria comenzaron a grabar Powerpoints con el contenido de una clase en un vídeo añadiéndole una explicación hablada. Este modelo de clase permitió desligar el seguimiento de una lección de lugar y tiempo. Pronto esta metodología flexible fue copiada y perfeccionada por otros. Para presentar contenidos en línea, resultó muy útil hacer uso de screencast, un software diseñado para explicar el funcionamiento de programas informáticos y que permitía grabar vídeos de una manera sencilla. Hoy en día, flipping the classroom es mucho más que sustituir una explicación clasical por un vídeo. Fundamentalmente se basa en emplear el tiempo de clase para profundizar en los aspectos teóricos que se han estudiado previamente fuera de clase. Mediante tareas de lectura o un vídeo explicativo grabado por el profesor u otra persona, el estudiante prepara los conceptos claves que, una vez dentro de la clase, trabaja de una forma activa, intercambiando conocimientos con sus compañeros y recibiendo retroalimentación de ellos y de su profesor. Para hacerlo puede usar la tecnología empleando el dispositivo que tenga a su alcance. Por tanto, BYOUD (Bring your own device, es decir, tráete tu propio dispositivo) es el concepto bajo el que permitiría al alumno trabajar con cualquier aparato tecnológico, bien sea un ordenador, una tableta o un teléfono móvil, o varios al mismo tiempo.

El flipped classroom y el blended learning tienen en común la presentación de contenidos fuera del aula. Sin embargo, donde el último puede ver reducidos los encuentros cara a cara a un mínimo (por ejemplo un encuentro de introducción y otro de cierre de un curso), el primero en un principio no contempla la reducción de los mismos. De ahí que está siendo introducido cada vez más en la educación secundaria para cambiar los modelos de clases tradicionales, pero manteniendo las horas de contacto.

1 El concepto recibió en un principio varios nombres. Salman Khan lo bautizó finalmente con el nombre de Flipped Classroom en una conferencia sobre el vídeo en la clase a través de Ted.com. Información extraída de http:/ / www.kennisnet.nl/themas/flipping-the-classroom/ [consultada el 23-3-2015]. 
Imagen 1, extraída de http://www.matt-koehler.com/tpack/ tpack-explained/ TPACK Image (rights free)

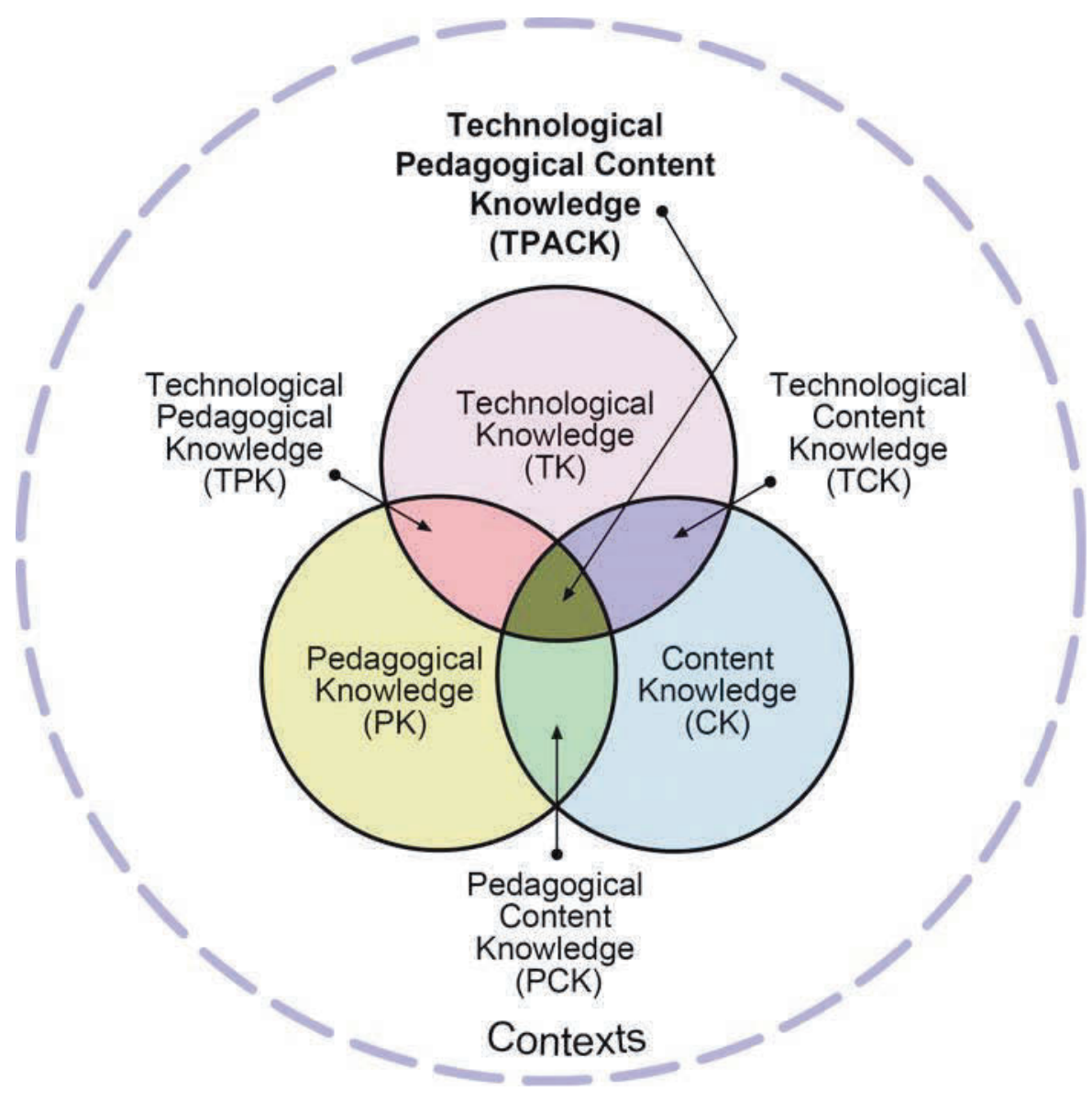

El modelo TPACK, (véase imagen 1) desarrollado por Koehler y Mishra, nos permite centrarnos en los conocimientos que necesita un docente para implementar las TIC el aula. Sirve de apoyo a la hora de diseñar una clase invertida. El modelo se centra en la intersección compleja de tres tipos de conocimiento, a saber, el conocimiento del Contenido (CK), el Pedagógico (PK) y el Tecnológico (TK) (véase imagen 1). Primeramente se verán los diferentes elementos por separado y a continuación su interrelación.

El conocimiento del contenido (CK) exige del docente conocer y dominar el tema que propone enseñar. Incluye conceptos, principios, teorías, ideas, procedimientos, puntos de vista, etc., sobre una materia. El conocimiento pedagógico (PK) alude a 
los conocimientos de procesos de enseñanza y aprendizaje, cómo trabajar los objetivos generales y específicos, la gestión del aula, las formas activantes de trabajo la planificación de las lecciones y la evaluación. El conocimiento tecnológico (TK) se refiere al conocimiento sobre los recursos tecnológicos y el uso de las herramientas. Se incluyen las tradicionales, como la pizarra o reproductores de $\mathrm{CD}$, pero también las modernas, como la pizarra digital, internet, vídeos digitales, las redes sociales, etc. El docente necesita de la comprensión de cómo aplicarlos de una manera productiva al trabajo y en la vida cotidiana, el reconocimiento de que pueden facilitar o entorpecer la consecución de un objetivo y la capacidad de adaptarse y renovarse permanentemente a los nuevos avances y versiones. Centrándose en los 4 espacios de intersección que generan sus interrelaciones se puede deducir que el conocimiento pedagógico del contenido (PCK) trata de la metodología empleada, es decir, la toma de decisiones que realiza el docente para presentar la materia en la manera que favorece mejor al aprendizaje del alumno. Para ello necesita adaptar los materiales didácticos disponibles, teniendo en cuenta los conocimientos previos del alumnado, el currículum, la programación y la evaluación.

El conocimiento tecnológico del contenido (TCK) se refiere a que el docente debe conocer las formas en que las tecnologías influyen de manera positiva o negativa en la presentación del contenido y saber qué tecnologías específicas son las más adecuadas para la enseñanza y el aprendizaje de según qué contenidos.

El conocimiento tecnológico pedagógico (TPK) alude a cómo repercuten las herramientas tecnológicas en la didáctica. Esto incluye el conocimiento de las ventajas y limitaciones de las distintas herramientas tecnológicas que pueden favorecer o limitar unas u otras estrategias pedagógicas.

Finalmente, el conocimiento tecnológico pedagógico del contenido (TPCK) une los cuatro elementos. Requiere una comprensión de las técnicas pedagógicas que usan tecnologías de forma constructiva para enseñar contenidos, de lo que facilita o dificulta el aprendizaje, de cómo se puede usar la tecnología para ayudar a resolver los problemas del alumnado, de la manera en que los alumnos aprenden usando tecnologías dando lugar a una construcción de conocimiento.

\section{SuPERANDO OBSTÁCULOS}

El metanálisis de unos 1.100 estudios sobre los efectos del aprendizaje en línea llevado a cabo por Means y otros concluye que las clases con un aprendizaje en línea, bien sea totalmente a distancia o de manera blended, producen de media mejores resultados del aprendizaje que las clases que son exclusivamente cara a cara. Esta conclusión resulta alentadora: adaptando las clases a un sistema de aprendizaje semipresencial ofrecerá la posibilidad de mejorar la calidad de la enseñanza. A esta ventaja se suman 
los ahorros en gastos de transporte y en tiempo de viaje y la flexibilidad de poder seguir las clases cuándo y dónde uno desee.

Sin embargo, todavía hay muchas dudas y preocupaciones acerca de la implementación de estos sistemas. Por parte de las instituciones y del profesorado existe la preocupación de no estar a la altura, sobre todo en cuanto a dispositivos y conocimientos de las TIC se refiere. Se teme que el ordenador sustituya al profesorado y no se tiene claro cómo afrontar el nuevo rol que se deduce, tanto de profesores como de alumnos. Ante esta incertidumbre y teniendo en cuenta los cambios que la sociedad impone, los expertos ${ }^{2}$ recomiendan dar pequeños pasos y no dejarse amedrentar.

Dado que hoy en día prácticamente todo el mundo se ve obligado a hacer uso de un ordenador en su actividad diaria, por no hablar del smartphone que se lleva en el bolsillo o la tableta que hay en casa, usar las TIC en el aula podría ser un obstáculo relativamente fácil de superar si se empieza por lo que se conoce e inspira tranquilidad, es decir, cuestionándose qué programas y aplicaciones utilizadas en la vida privada serían de utilidad en el aula. Al mismo tiempo convendría trabajar en la competencia digital propia y reflexionar cómo reforzarla tanto personal como profesionalmente, sirviéndose de compañeros de trabajo, revistas (digitales), páginas web, vídeos, foros, conferencias, blogs, etc.

Modificar la clase hacia un modelo de B-learning ofrece la libertad de replantear los materiales y contenidos que se trabajan, cuestionar la presentación, la ordenación y la utilidad de los mismos. Quizás conviene omitir ciertos aspectos de los contenidos que se han quedado obsoletos y sustituirlos por otros cuyo descubrimiento o actualidad los han convertido en tratamiento obligatorio en las aulas. Encontrar material digitalizado acerca de ellos no resultará demasiado difícil, pero se necesitará del ojo crítico de un profesor para discriminar la relevancia didáctica y su adaptación a las necesidades del alumno. De ahí cabe deducir que el profesor continuará siendo un elemento clave en la enseñanza del futuro, puesto que sus conocimientos pedagógicos y metodológicos seguirán siendo imprescindibles en la adecuación de contenidos para el aula.

Donde el profesor antes era el centro de atención de una clase, al que todo alumno debería escuchar durante largo tiempo, con el modelo de blended learning, aquel se transforma en un coach, es decir, la persona que acompaña y guía al alumno en el proceso de aprendizaje. El alumno, a su vez, pasará de ser un consumidor de contenidos a un agente activo. Y para poder desempeñar ese papel, necesita de alguien que le ofrezca tareas activantes, que le hagan poner en práctica los conocimientos

2 Means (2011) recomienda seguir contando en clase lo que no se puede presentar de otro modo. 
previamente presentados con el fin de llegar a un nivel de mayor profundización de conocimiento. He aquí el nuevo rol del profesor.

El reto que se plantea es cómo conseguir estimular la motivación intrínseca del alumno de tal manera que le lleve a prepararse los materiales ofrecidos fuera del aula. El punto de partida serían los conocimientos y habilidades previos que presentan los estudiantes. Conociendo estos se puede hacer una diferenciación entre lo que se puede contar o dejar leer - por tratarse de mera información- y lo que necesita de un aprendizaje más profundo ${ }^{3}$ y requiere de otros medios, para, por ejemplo, automatizar procedimientos o reglas, contrastar casuísticas, o desarrollar una actitud crítica.

Sea cual sea el objetivo a cumplir, conviene especificarlo y determinar los pasos para llegar a su realización, dejándolos claros no solo para el profesor, sino también para el alumno, y haciéndole así responsable de su proceso de aprendizaje. Sobre todo al principio del aprendizaje conviene ofrecer al estudiante la posibilidad de planificar y revisar lo aprendido y reflexionar en torno a ello en una estructura predeterminada y con unas claves prefijadas. A medida que vayan progresando, el control se va dejando en manos de los alumnos.

Todo lo que suscita curiosidad, entusiasmo y ofrece satisfacción, hace que se aprenda más. Teniendo en cuenta esto, convendría contar con estos elementos afectivos a la hora de preparar los materiales. El estudio de Means refleja que gran parte del éxito del B-learning se debe a la interactividad ${ }^{4}$ que ofrece: a más interacción entre el profesor y el alumno o entre el alumno y el material, mejores resultados académicos. Si además hay más interacción entre peers, es decir, sus compañeros, los cursos se valoran mejor. Por tanto, conviene ofrecer actividades colaboradoras que apoyen el aprendizaje de compañeros más expertos. Para ello se podrían diseñar tareas que obliguen al intercambio de información, lleven a la resolución de casos y estimulen la creatividad y la reflexión.

Es recomendable que se empleen diferentes formas de evaluación tanto sumativas como formativas. Estas últimas deberían incluir la autocorrección e integrarse preferiblemente en las actividades en línea para que el alumno le vea la utilidad. A la hora de idear maneras de evaluar los contenidos, es de gran utilidad la taxonomía de Bloom digital que permite ver cómo llevar a cabo el aprendizaje mediante diferentes herramientas digitales en función de la capacidad cognitiva que se requiere.

3 Se podría hacer la distinción entre información (information/moderately new knowledge), conocimientos y habilidades (procedures/skills) y aprendizaje profundo o construcción de conocimientos (deeper learning / knowledge building). Means (2011)

4 Means (2011), citando un estudio de Robert Bernard y otros sobre tipos de tratamiento de la interacción en la educación a distancia. 


\section{CONCLUSIONES}

B-learning ofrece la posibilidad de encaminar la enseñanza a las exigencias del siglo XXI. Para ello se necesita de una inversión personal, profesional e institucional de las TIC. No requiere de grandes sacrificios, pero sí de la voluntad de querer progresar hacia un tipo de enseñanza centrado en el individuo. Para que el alumno aprenda a cooperar, negociar, desarrollar una actitud crítica y crear, destrezas ineludibles en este siglo, harán falta profesores que les guíen. El profesor no perderá importancia: su presencia dejará de estar delante de la clase a ocupar un lugar entre los alumnos, bien sea ayudándoles en sus tareas en el aula o haciendo el seguimiento de sus avances a través de un dispositivo electrónico. El primer paso será entender la terminología que va con ello. Que esta reflexión sirva para ello.

\section{Bibliografía}

Fransen, J., (2006). "Een nieuwe werkdefinitie van blended learning". OnderwijsInnovatie, juni 2006, 26-29. Koehler, M. J., y Mishra, P. (2009). "What is technological pedagogical content knowledge?" Contemporary Issues in Technology and Teacher Education, volume 9 (1), 60-70.

Means, B. et.al. (2009). Evaluation of Evidence-Based Practices in Online Learning: A Meta-Analysis and Review of Online Learning Studies, U.S. Department of Education, Office of Planning, Evaluation, and Policy Development Washington D.C., Disponible en: http://www2.ed.gov/rschstat/eval/tech/evidence-based-practices/finalreport.pdf [fecha de consulta: 20-3-2015]

Means, B. (2012) Blended Learning Designs: A Learning Science Perspective, ELI annual Meeting, podcast disponible en: http://www.educause.edu/eli/events/eli-annual-meeting/2011/blendedlearning-designs-learning-science-perspective [fecha de consulta: 16-3-2015]

Oliver, M. y Trigwell, K. (2005), “Can 'Blended Learning' be redeemed?” E-Learning and Digital Media, Volume 2, (1), 17-26.

VV.AA., Vier in balans monitor 2013, Kennisnet, Zoetermeer, Disponible en: www.kennisnet.nl/ onderzoek/vier-in-balans-monitor/ [fecha de consulta: 15-3-2015]

VV.AA. A guide for using Bloom's taxonomy in blended learning. Disponible en: http://www.uws.edu.au/_data/assets/pdf_file/0008/452078/Blooms_taxonomy_for_blended_learning.pdf [fecha de consulta: 27-3-2015]

Website sobre flipping the classroom, su historia y teoría, disponible en: http:/ /www.kennisnet.nl/themas/flipping-the-classroom/ [fecha de consulta: 15-3 a 28-3-2015]

ana.llamazares@hu.nl 